\title{
Drawing Road Networks with Mental Maps
}

\author{
Shih-Syun Lin, Chao-Hung Lin, Member, IEEE, Yan-Jhang Hu, and Tong-Yee Lee, Senior Member, IEEE
}

\begin{abstract}
Tourist and destination maps are thematic maps designed to represent specific themes in maps. The road network topologies in these maps are generally more important than the geometric accuracy of roads. A road network warping method is proposed to facilitate map generation and improve theme representation in maps. The basic idea is deforming a road network to meet a user-specified mental map while an optimization process is performed to propagate distortions originating from road network warping. To generate a map, the proposed method includes algorithms for estimating road significance and for deforming a road network according to various geometric and aesthetic constraints. The proposed method can produce an iconic mark of a theme from a road network and meet a user-specified mental map. Therefore, the resulting map can serve as a tourist or destination map that not only provides visual aids for route planning and navigation tasks, but also visually emphasizes the presentation of a theme in a map for the purpose of advertising. In the experiments, the demonstrations of map generations show that our method enables map generation systems to generate deformed tourist and destination maps efficiently.
\end{abstract}

Index Terms-Map generation, mesh warping, information visualization

\section{INTRODUCTION}

$\mathrm{E}$ XISTING maps can be classified into two categories: topographic maps and thematic maps. Topographic maps are constructed for general audiences and thus contain varied map elements and information. By contrast, thematic maps are particularly designed to display a specific theme of an area. Topographic maps accurately display where something is located in a space, whereas thematic maps focus on combining specific themes with maps. With the development of information visualization techniques, thematic maps have drawn increasing attention in the fields of visualization and computer graphics. Several excellent systems for thematic map generation have recently been proposed [1], [2], [3]. These systems automatically generate tourist and destination maps to facilitate route planning and navigation in unfamiliar areas. These systems emphasize important roads and landmarks while de-emphasizing less important elements in a map by using cartographic generalization techniques. The proposed algorithm can draw a road network that not only emphasizes important map elements but also presents the icons of themes in road networks for the purpose of advertising.

The geometric shape accuracy of map elements is generally less important than road network topologies, and road networks are sometimes simplified and deformed to improve visual representation. For instance, studies on route map generation search for an optimal route layout with the correct topology [1], [4], [5], [6]. Routes in maps are

- S.-S. Lin, Y.-J. Hu, and T.-Y. Lee are with the Department of Computer Science and Information Engineering, National Cheng Kung University, Tainan 701, Taiwan, R.O.C. E-mail: catchylss@hotmail.com, qwas368@gmail.com, tonylee@mail.ncku.edu.tw.

- C.-H. Lin is with the Department of Geomatics, National Cheng Kung University, Tainan 701, Taiwan, R.O.C.E-mail: linhung@mail.ncku.edu.tw.

Manuscript received 24 July 2013; revised 14 Feb. 2014; accepted 26 Feb. 2014. Date of publication 16 Mar. 2014; date of current version 30 July 2014.

Recommended for acceptance by $\mathrm{G}$. Andrienko.

For information on obtaining reprints of this article, please send e-mail to: reprints@ieee.org, and reference the Digital Object Identifier below.

Digital Object Identifier no. 10.1109/TVCG.2014.2312010 not required to have the same shapes and lengths as those of actual routes. Another example is shown in Fig. 1. A map containing a guitar shape in the road network of London is created by an artist. Abbey Road is famous for the Beatles album of the same name. For this reason, this place has become a tourist attraction; the map of this area can serve as a tourist map not only for navigation but also for advertising. Similarly, in Fig. 2, a deformed map is created for better representation of tourist attractions. These two examples show that tourist and destination maps are designed not only for navigation, but sometimes for attracting customers and tourists. A tourist or destination map that contains approximate locations of destinations or tourist attractions is sufficient for tourists and customers because they do not really navigate the landscape. However, large curvatures of a route map are often used as navigational cues in the cases of walking and driving. A map with significant deformation may lose the navigation utility. Our user study also indicates that user satisfaction decreases, in terms of map navigation, with increasing map deformation. Therefore, in the proposed scheme, the road network is deformed according to the mental map specified by users through a simple interface. If an accurate navigation is required, a large deformation must be avoided in the design of the mental map. By contrast, if advertising is the main purpose, a large deformation is allowed.

To facilitate the generation of deformed tourist and destination maps, a road network warping method is proposed. In the proposed warping, a grid mesh is created to cover the road network, and the grid mesh is then deformed according to various geometric and aesthetic constraints. In warping, high-significance roads are forced to undergo as-rigidas-possible deformation by shape-preserving constraints while smoothly propagating distortions through an optimization process. A smoothing constraint is also introduced in the optimization to reduce the possibility of mesh foldover (mesh flipping) and to fit the user-specified mental map in warping. With the aid of various constraints and road significance measurements, an iconic mark can be produced 

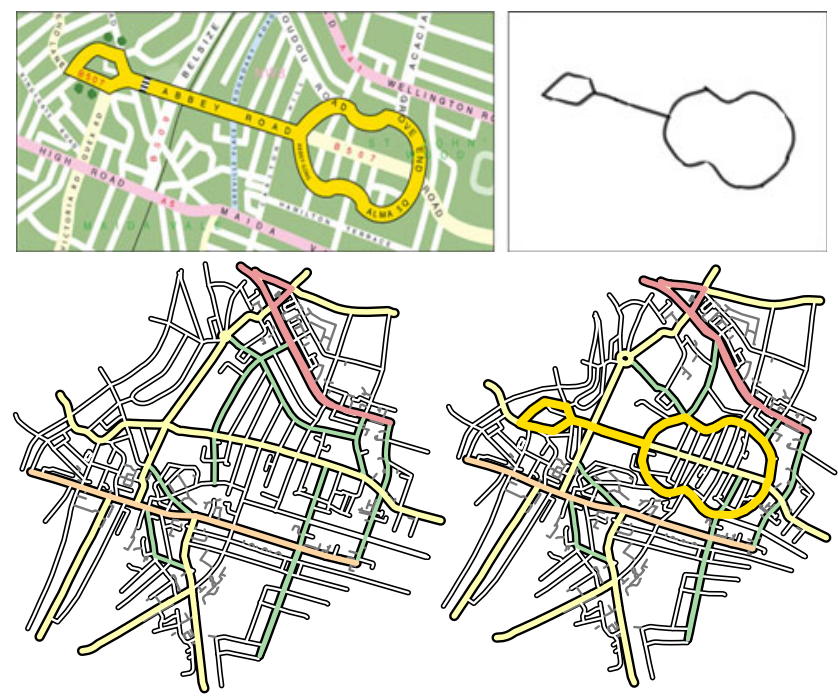

Fig. 1. Map generation by using the proposed method. Top: artistdesigned map (left) and mental map (right); bottom: original road network of London (left) and our result (right).

from a road network (Fig. 1) and users can stylize their own maps (Fig. 17). Therefore, our method can enable map generation system to create various deformed thematic maps efficiently according to user-specified mental maps and road significance measurements.

\section{Related Work}

Previous studies on the generation of deformed maps, including tourist and destination maps, are discussed in this section. For topographic maps, a detailed survey can be found in [7]. Generally, the requirements of fixed-scale and accurate representation of visual elements in topographic maps are unsuitable for deformed maps because important roads and regions may be invisible in a fixed-scale map which contradicts the generalization principles of deformed maps. Therefore, warping techniques are used in the deformed map generation. A naïve method is to apply existing warping techniques to enlarge and emphasize important regions of a fixed-scale map [5], [8], [9]. However, the unnatural distortions of road networks make route planning and navigation difficult for users. To solve this problem, researchers have devoted their efforts to map warping.

In the last decade, many road network warping methods have been proposed for metro map generation. The routes are usually drawn as straight lines, and the lines vary in direction with only a few fixed angles (e.g., 45 and 90 degrees). Therefore, drawing a metro map for a given road network is referred to line simplification and layout optimization, which have been studied extensively in the fields of map generation and information visualization [5], [10], [11], [12].

In the work of Agrawala and Stolte [1], an elegant cartographic generalization method is introduced to improve the usability of route maps. Their method is based on the analysis of hand-drawn route maps and cognitive psychology research shows that the exact length, angle, and shape of each road are rather unimportant [13]. By adopting nonlinear optimization, the road network is rearranged in a way

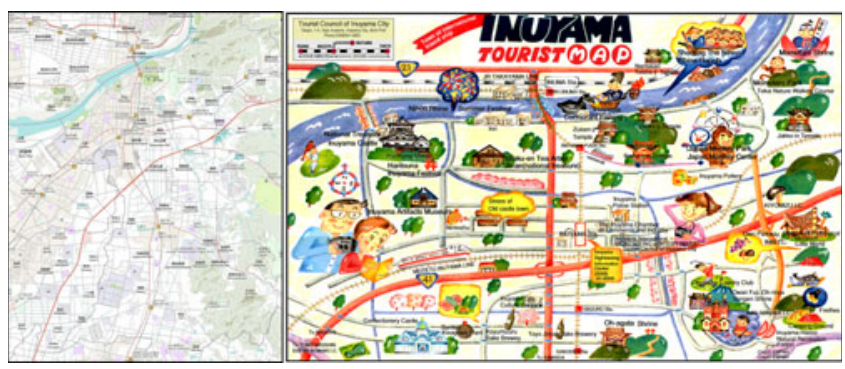

Fig. 2. Deformed tourist map. Left: original map; right: artist-designed tourist map. The tourist map is deformed for better representation.

that makes the result similar to a hand-drawn map. Similarly, Kopf et al. [3] propose a system to create destination maps based on the cognitive psychology of map designs [14] and cartographic generalization [15]. This system includes techniques for selecting important roads on the basis of mental representations of road networks and for laying out roads by using a non-linear optimization procedure. The proposed method, which is similar to the method proposed in [3], involves a non-linear road network warping that not only preserves the shape and topology of a road network but also forces a road network to meet user-defined constraints. Thus, our approach can drive the generalization of various deformed maps and improve the representation of a specific theme for advertising.

Grabler et al. [2] propose a tourist map generation system wherein non-photorealistic renderings of 3D models are used as map elements to emphasize important landmarks and points of interest while irrelevant elements are de-emphasized or eliminated. This system can yield good results but requires a complex geometrical and textured 3D city model, thus making the system infeasible for map generation. Similarly, Simon et al. [16] and Chen et al. [17] present novel methods that generate image icons to represent popular landmarks in a map. Inspired by the concept of photo tourism [18], they synthesize or generate an image icon from a set of geotagged photos for landmark presentation. However, image icon generation requires the nontrivial processes of image matching and warping. In comparison, the warping method in the current study has the ability to produce a representative iconic mark of tourist attractions from the road network. Therefore, the resulting map can provide a good visual representation of a tourist theme in a map.

Isenberg [19] and Afzal et al. [20] have recently addressed aesthetic map rendering and map art generation. Isenberg [19] adopts the techniques of map simplification, abstraction, displacement, and shading to generate maps with the goal of producing aesthetic renderings of map elements. Afzal et al. [20] introduce an algorithm to generate a map by using only text as the graphical feature. Therefore, providing the functions of map navigation and route planning are no longer the goals of map art generation. With the aid of our warping approach, artists can use these two methods [19], [20] to generate elegant map arts with the proposed road network deformation.

Our method relies on warping and least-squares optimization, which have been studied in related areas including focus+context visualization [21], [22], content-aware 
image/video retargeting [23], and map conflation [24], [25]. In focus+context visualization, a user-defined focus region is enlarged by minimizing a defined distortion function. In content-aware retargeting, images or videos are resized to fit displays with various aspect ratios while preserving visually salient content. The problems in these two topics are generally formulated as least-squares optimization problems wherein important and focus regions are preserved or even enlarged and distortions are propagated to homogenous regions. Similarly, in the current study, the local shape of a road network is preserved as much as possible while distributing the distortion to neighboring regions. In map conflation, the geographic information of maps from overlapping sources is integrated into a richer data set. After data matching, a warping method that uses least-squares optimization [24], [25] is adopted to fuse the geometric information from different sources. To preserve the geometric shapes in overlapping regions, a rigid transformation constraint is introduced in the optimization. The deformation problems are formulated as least-squares optimizations in the current method and these related methods. However, different purposes require different objective functions and processes. The objective functions in [21], [22], [24], [25] do not contain user-specified constraints and road significance measurements which are necessary for the fitting of the mental map and the preservation of highly significant roads in the proposed scheme.

\section{Methodology}

The proposed method consists of three main steps: user input, significance measurement, and road network warping. The input to our method is a user-specified mental map and a road network represented by vector graphics. A road network is described by a triple $\mathbf{R N}=(\mathbf{N}, \mathbf{H}, \mathbf{R})$, where $\mathbf{N}=\left\{N_{1}, \ldots, N_{n_{\text {node }}}\right\}$ represents the positions of nodes in the road network, $\mathbf{H}=\left\{H_{1}, \ldots, H_{n_{h}}\right\}$ is a set of line segments that represent the connectivity of the road network, and $\mathbf{R}=\left\{R_{1}, \ldots, R_{n_{r}}\right\}$ is a set of roads, in which each road is represented by a set of connected line segments in $\mathbf{H}$. Here, $n_{\text {node }}, n_{h}$, and $n_{r}$ represent the number of nodes, line segments, and roads, respectively. In addition, a mental map that contains a mapping $\left\{\left(G_{i}, N_{m(i)}\right)\right\}_{i=1}^{n_{g}}$ between the user-specified road nodes $G_{i}$ and the nodes in the input road network $N_{m(i)}$ is provided by users through an interface, where $n_{g}$ represents the number of user-specified nodes, i.e., geometric constraints, and $m$ is the index mapping function. Note that we focus on the road network drawing with a mental map rather than the sketch-based user interface. Therefore, a simple interface with road selection and line drawing functions is provided for users to define the mapping between the mental map and input road network. Please refer to the accompanying videos for more details on the user interface.

In the significance measurement, we propose a roadbased measurement that assigns a significance value to a road such that the roads can be deformed as consistently as possible. Moreover, the connectivity of the road network is considered in the significance measurement. In the last step, a grid mesh is created to cover the road network. Thereafter, the proposed warping is performed to force the highsignificance roads and quads to undergo as-rigid-as-possible deformation during warping. The significance measurement is introduced in Section 3.1 followed by road network warping, which is presented in Section 3.2.

\subsection{Significance Measurement}

The basic idea of our method is to preserve the geometric shapes of high-significance roads and quads, and propagate distortions to low-significance regions. Therefore, we measure the significance of roads and quads in a preprocessing step. Following the road level defined in the general traffic systems [26] and the principles in the cartographic generalization that creates contrast between high-level and low-level roads [14], we assign the significance values of roads according to their levels in the traffic system. This means that the highest value (i.e., 1.0) is assigned to the most significant roads, such as national freeways, and the lowest value (i.e., 0.1) is assigned to the least significant roads, such as lanes. Thus, high-level roads have better shape preservation than low-level roads in warping. In addition, according to the cognitive psychology of map design [27], a high significance value is assigned to a road with regular shape (e.g., straight line and circle) because people can more easily perceive deformation from regular shapes than deformation from irregular shapes. In the implementation, the shape of the road is represented by the radii of the discrete curvatures of road nodes. Given a node $N_{j}$ and its neighboring nodes $N_{j+1}$ and $N_{j-1}$, the radius of the discrete curvature of node $N_{j}$ is defined as the vector angle $\angle N_{j-1} N_{j} N_{j+1}$ :

$$
C\left(N_{j}\right)=\arccos \left(\frac{\left(N_{j-1}-N_{j}\right) \cdot\left(N_{j+1}-N_{j}\right)}{\left\|N_{j-1}-N_{j}\right\|\left\|N_{j+1}-N_{j}\right\|}\right) .
$$

The shape regularity of a road is then measured by the standard deviation of radii of node curvatures:

$$
\operatorname{Reg}\left(R_{i}\right)=\sqrt{\frac{1}{n_{R i}} \sum_{N_{j} \in R_{i}}\left(C\left(N_{j}\right)-\overline{C\left(N_{R_{i}}\right)}\right)^{2}},
$$

where $n_{R i}$ represents the number of nodes in the road $R_{i}$, and $\overline{C\left(N_{R_{i}}\right)}$ is the mean of the radii. A significance value is calculated and assigned to each road by combining these two factors (i.e., road level and shape regularity):

$$
\operatorname{Sig}\left(R_{i}\right)=\left(N \operatorname{Reg}\left(R_{i}\right)+N \operatorname{Level}\left(R_{i}\right)\right) / 2,
$$

where $N \operatorname{Reg}\left(R_{i}\right)$ and $N \operatorname{Level}\left(R_{i}\right)$ represent the normalized shape regularity and normalized road level of the road $R_{i}$, that is, $N R e g \in[0.1,1.0]$ and $N$ Level $\in[0.1,1.0]$. Note that any other considerations on the road significance measurement, such as the user-specified significance values and road selection strategies used in [28], can be integrated in the proposed scheme.

The connectivity of the road network is considered in the quad significance measurement. The quad significance is defined as the summation of valences (or degrees) of road nodes within the quad face. The valence of a node is the number of edges incident to the node. This definition can preserve the geometric relations of 


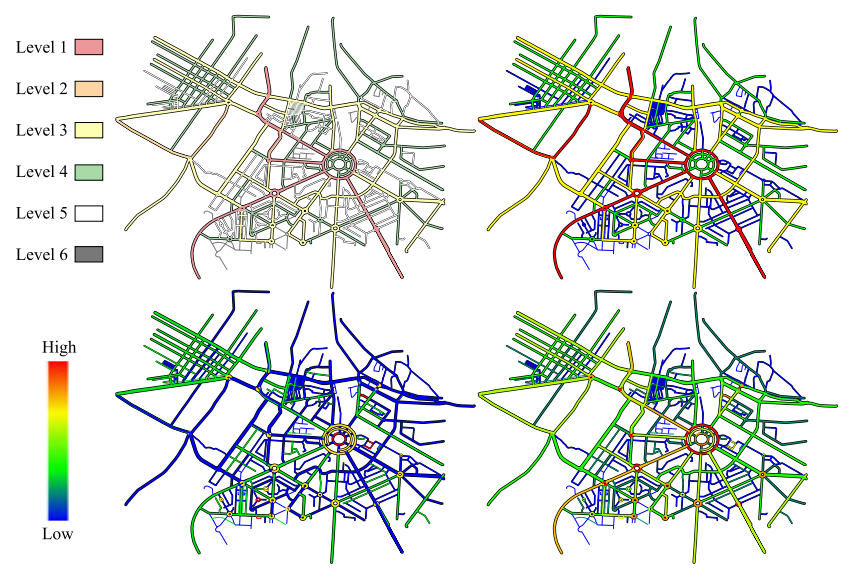

Fig. 3. Road significance measurement. Top: original road network containing six road levels (left) and significance value of road level (right); bottom: significance value of shape regularity (left) and combined significance value (right). The significance values are visualized by colors ranging from blue (lowest value) to red (highest value).

roads that intersect at a high-valence or high-significance node (i.e., the intersection of roads). Note that only the road nodes are considered in the significance measurement because road length is less important than the geometric relations between road nodes, and ignoring the preservation of the road length can increase the flexibility of the map warping. Moreover, user-specified focus regions and other map elements, such as coastlines and rivers, can be further considered in the significance measurement. Figs. 3 and 4 show examples of road and quad significance measurement, respectively. Obviously, highlevel roads with regular shapes are assigned high significance values, and quads that contain high-valence intersections are assigned high significance values. Thus, the proposed significance measurement can aid the warping method in preserving the shapes and geometric relations of high-significance roads.

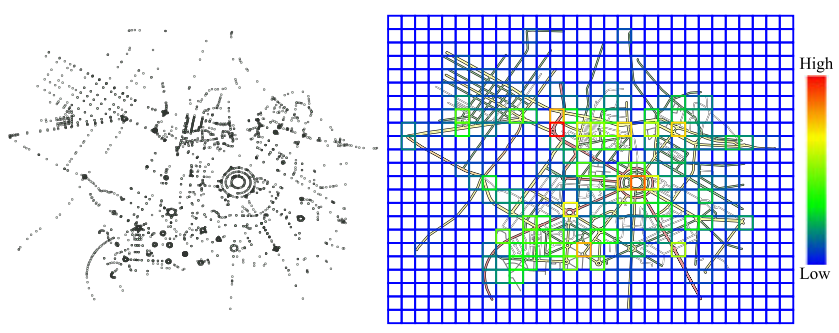

Fig. 4. Quad significance measurement. Left: road nodes; right: valences of nodes and significance values of quads. The significance values are visualized by colors ranging from blue (lowest value) to red (highest value).

\subsection{Feature-Preserving Road Network Warping}

In creating tourist or destination maps, designers generally distort road geometry but preserve road topology. Therefore, a warping method with a control mesh defined over a map is generally used in map generation. In [22], the road network is used as a control mesh in warping under the assumption that the network is connected. For an unconnected network, some virtual roads or edges are inserted to make the network connected. A triangle mesh built upon a road network is another solution. However, a road generally occupies several triangles, and the inconsistent orientations of deformed triangles may result in inconsistent warping on roads. For example, in Fig. 5f, inconsistent warping occurs in the area marked by red quadrangle wherein the orientations of the deformed triangles are inconsistent. The inconsistent orientations are mainly caused by the irregular structure of the triangle mesh. In the current study, we propose the use of a regular grid mesh as the control mesh instead of an irregular triangle mesh, which potentially provides better warping in terms of consistency of road deformation, as shown in Fig. 5.

Initially, a grid mesh $\mathbf{M}=(\mathbf{V}, \mathbf{E}, \mathbf{Q})$ that contains a vertex set $\mathbf{V}=\left\{V_{1}, \ldots, V_{n_{v}}\right\}$, an edge set $\mathbf{E}=\left\{E_{1}, \ldots, E_{n_{e}}\right\}$, and a quad set $\mathbf{Q}=\left\{Q_{1}, \ldots, Q_{n_{q}}\right\}$ is created to cover the

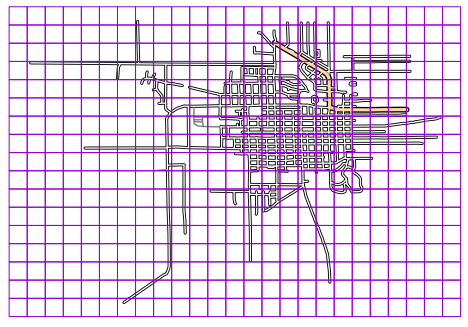

(a)

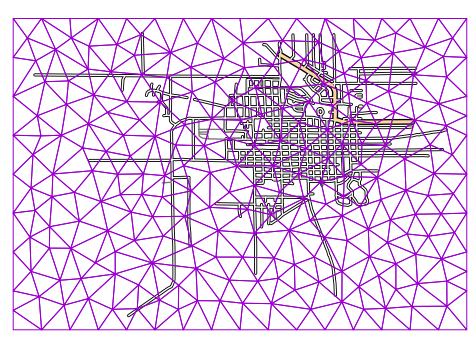

(d)

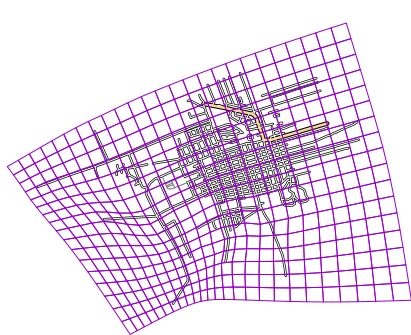

(b)

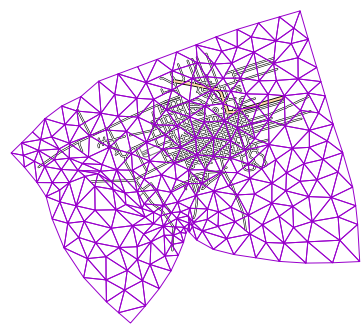

(e)

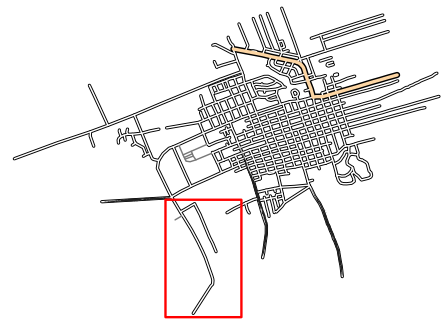

(c)

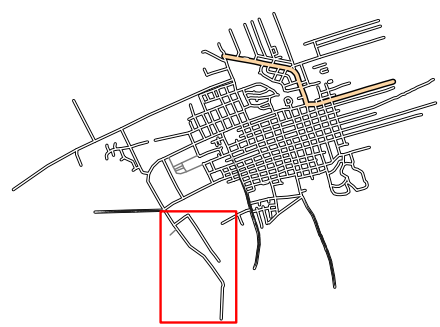

(f)

Fig. 5. Warping using grid mesh and triangle mesh. (a) Grid mesh; (b) deformed grid mesh; (c) result of grid mesh warping; (d) triangle mesh; (e) deformed triangle mesh; (f) result of triangle mesh warping. 


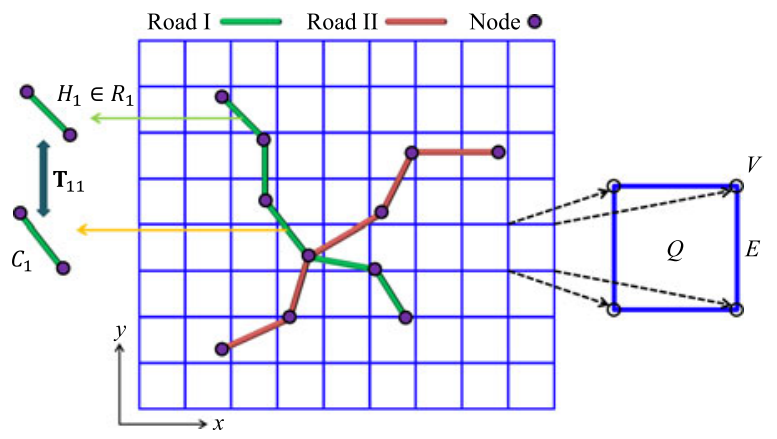

Fig. 6. Illustration of grid mesh and road preservation. $C_{1}$ is the representative line segment of the road $R_{1} ; \mathbf{T}_{11}$ is the transformation matrix between the line segments $H_{1}$ and $C_{1} . V$ and $E$ represent the vertex and the edge of the quad face $Q$.

original road network (Fig. 6), where $n_{v}, n_{e}$, and $n_{q}$ represent the number of vertices, edges, and quads, respectively. In addition, several pairs of corresponding nodes (i.e., geometric constraints) are provided by users, that is, $\left\{\left(G_{1}, N_{m(1)}\right), \ldots,\left(G_{n_{g}}, N_{m\left(n_{g}\right)}\right)\right\}$. The goal is to warp the road network to meet the user-defined constraints while preserving the shape and topology of the road network. Thus, we aim to find a deformed grid mesh and a deformed vertex set $\tilde{\mathbf{V}}=\left\{\tilde{V}_{1}, \ldots, \tilde{V}_{n_{v}}\right\}$, in which highsignificance roads and quads are deformed as rigidly as possible, and the distortions are propagated to the low-significance regions while preserving the topology of the road network. To achieve this goal, three energies, namely, shape-preserving, user control, and smoothing, are introduced to the road network warping with an optimization solver. These three energies are described below.

Shape-preserving energy. To preserve high-significance regions and roads and avoid inconsistent deformation on significant roads, two energy terms are included in the optimization, namely, road-preserving and quad-preserving. To calculate the road-preserving energy, a representative line segment is selected for each road. The line segment containing the middle of the road is selected as the representative line segment. Given an arbitrary line segment $H=\left(N_{a}, N_{b}\right)$ in the road, the geometric transformation $\mathbf{T}$ between the line segment $H$ and representative line segment, which is denoted by $C=\left(N_{m}, N_{n}\right)$, can be formulated as follow:

$$
\begin{aligned}
H=\mathbf{T} C & \Rightarrow\left[\begin{array}{l}
H_{x} \\
H_{y}
\end{array}\right]=\left[\begin{array}{cc}
s & r \\
-r & s
\end{array}\right]\left[\begin{array}{l}
C_{x} \\
C_{y}
\end{array}\right] \\
& \Rightarrow\left[\begin{array}{l}
s \\
r
\end{array}\right]=\left[\begin{array}{cc}
C_{x} & C_{y} \\
C_{y} & -C_{x}
\end{array}\right]^{-1}\left[\begin{array}{l}
H_{x} \\
H_{y}
\end{array}\right],
\end{aligned}
$$

where

$$
\left\{\begin{array} { l } 
{ C _ { x } = N _ { m _ { x } } - N _ { n _ { x } } } \\
{ C _ { y } = N _ { m _ { y } } - N _ { n _ { y } } }
\end{array} \text { and } \left\{\begin{array}{l}
H_{x}=N_{a_{x}}-N_{b_{x}} \\
H_{y}=N_{a_{y}}-N_{b_{y}}
\end{array}\right.\right.
$$

and the suffixes $x$ and $y$ represent the $x$ - and $y$-components of the line segment and node position. To address the problem of inconsistent deformation on roads, the road-preserving energy, denoted by $D_{\text {RoadP, }}$, is formulated as follows to measure the rigidity of the road by using the selected representative line segment $H$ and corresponding geometric transformation $\mathbf{T}$ :

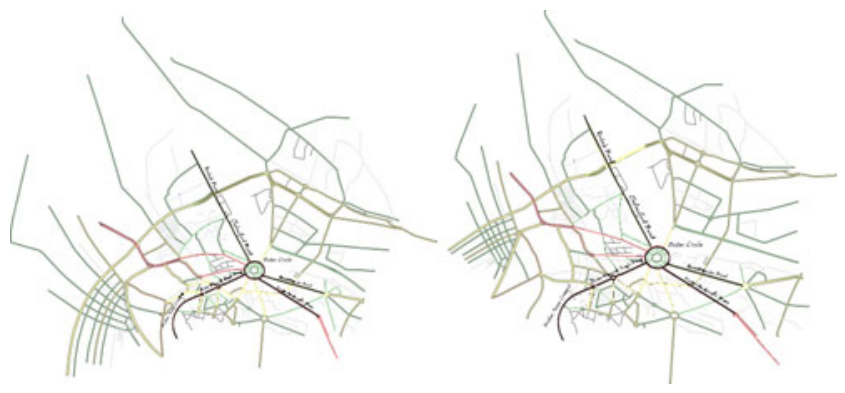

Fig. 7. Comparison between warping with (right) and without (left) the road-preserving energy term. The original road network and road significance values are shown in Fig. 3.

$$
D_{\text {RoadP }}(\mathbf{R N})=\sum_{i=1}^{n_{r}} s_{i} \times \sum_{H_{j} \in R_{i}}\left\|\tilde{H}_{j}-\mathbf{T}_{i j} \tilde{C}_{i}\right\|^{2},
$$

where $s_{i}$ is the significance value of the road $R_{i}$, and its range is $[0.1,1.0] ; \tilde{H}_{j}$ and $\tilde{C}_{i}$ represent the deformed line segment and deformed representative line segment of the road $R_{i}$, respectively; $\mathbf{T}_{i j}$ represents the geometric transformation between $H_{j}$ and $C_{i}$ (Fig. 6). Therefore, the roadpreserving energy measures the changes of geometric relations in line segments that belong to a road, and a highsignificance road has high consistency on the deformations of its line segments. Assume that the nodes of line segments $H_{j}:\left\{N_{a}^{H_{j}}, N_{b}^{H_{j}}\right\}$ and $C_{i}:\left\{N_{m}^{C_{i}}, N_{n}^{C_{i}}\right\}$ lie within the quad faces $\left\{V_{p}^{H 1}\right\}_{p=1}^{4},\left\{V_{p}^{H 2}\right\}_{p=1}^{4},\left\{V_{q}^{C 1}\right\}_{q=1}^{4}$, and $\left\{V_{q}^{C 2}\right\}_{q=1}^{4}$, respectively. These nodes can be rewritten as a linear combination of quad vertices by using barycentric coordinate. Thus, Eq. (5) is reformulated as follows:

$$
\begin{aligned}
D_{\text {RoadP }}(\mathbf{M})= & \sum_{i=1}^{n_{r}} s_{i} \times \sum_{H_{j} \in R_{i}} \|\left(\sum_{p=1}^{4} b_{p}^{1} \tilde{V}_{p}^{H 1}-\sum_{p=1}^{4} b_{p}^{2} \tilde{V}_{p}^{H 2}\right) \\
& -\mathbf{T}_{i j}\left(\sum_{q=1}^{4} b_{q}^{1} \tilde{V}_{q}^{C 1}-\sum_{q=1}^{4} b_{q}^{2} \tilde{V}_{q}^{C 2}\right) \|^{2},
\end{aligned}
$$

where $b$ s denote the coefficients of the barycentric coordinates of the quad vertices $V \mathrm{~s}$.

Similarly, to preserve the road intersections lying within quads, the quad-preserving energy, denoted by $D_{Q u a d P}$, is formulated as follows to measure the rigidity of quads in warping:

$$
D_{Q u a d P}(\mathbf{M})=\sum_{i=1}^{n_{q}} g_{i} \times \sum_{E_{j} \in Q_{i}}\left\|\tilde{E}_{j}-\mathbf{U}_{i j} \tilde{K}_{i}\right\|^{2},
$$

where $g_{i}$ is the quad significance value; $\tilde{E}_{j}$ and $\tilde{K}_{i}$ represent the deformed edge and deformed representative edge of the quad $Q_{i}$, respectively. The top edge in a quad is selected as the representative edge. $\mathbf{U}_{i j}$ is the geometric transformation between $E_{j}$ and $K_{i}$. Therefore, the changes of geometric relations in quad edges are measured by this energy, and a high-significance quad has a high consistency on the deformation of quad edges. The total shape-preserving energy, denoted by $D_{S P}$, is defined by summing up the individual energy term as follows:

$$
D_{S P}(\mathbf{M})=D_{\text {RoadP }}(\mathbf{M})+D_{\text {QuadP }}(\mathbf{M}) .
$$




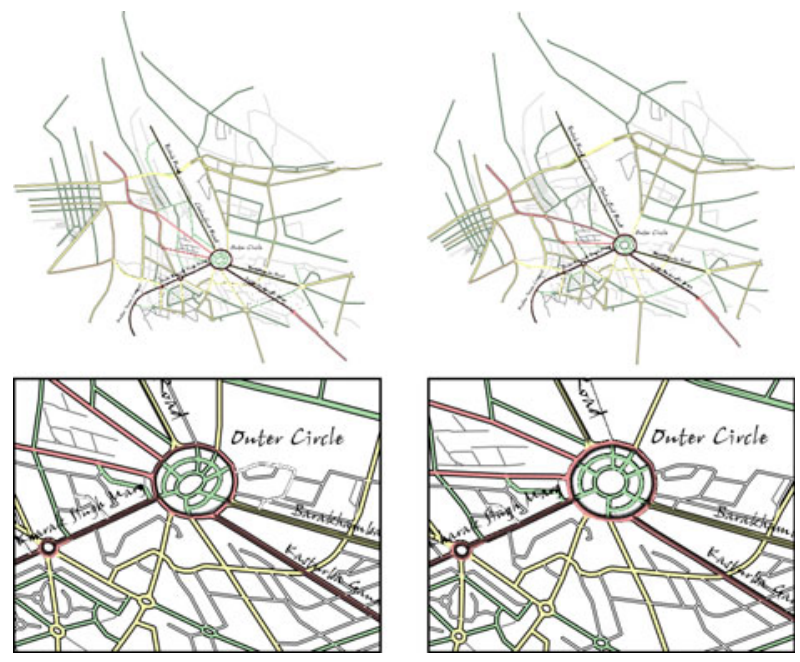

Fig. 8. Comparison between warping with (right) and without (left) quadpreserving energy term. The subfigures shown in the bottom are the close-up views of the warping results shown in the top. The original road network and quad significance values are shown in Figs. 3 and 4 , respectively.

To demonstrate the usability of the road-preserving and quad-preserving energies, warpings with and without these two energies are tested. The results are shown in Figs. 7 and 8. In Fig. 7(left), some roads with regular shapes are distorted after the warping. The human visual system is sensitive to this distortion. By contrast, with the aid of the road-preserving term, the shapes of highsignificance roads are efficiently preserved, as shown in Fig. 7(right). Similarly, some circular intersections are preserved by using the quad-preserving term, as shown in Fig. 8.

User control energy. The proposed method allows users to define constraints on a road network by sketching roads and specifying the correspondence between the sketches and the road network. An energy term called control energy is defined to force the road network to meet the user-defined constraints in warping. The control energy, denoted by $D_{U C}$, is defined as measuring the distances between the user-specified positions of nodes $G$ and the positions of their corresponding nodes $N$ in the deformed road network:

$$
D_{U C}(\mathbf{R N})=\sum_{i=1}^{n_{g}}\left\|\tilde{N}_{i}-G_{m(i)}\right\|^{2} .
$$

Similarly, Eq. (9) is reformulated by using barycentric coordinates given by the following:

$$
D_{U C}(\mathbf{M})=\sum_{i=1}^{n_{g}}\left\|\sum_{p=1}^{4} b_{i_{p}} \tilde{V}_{i_{p}}-G_{m(i)}\right\|^{2}
$$

where $b_{i_{p}}$ is the barycentric coefficients of $N_{i}$.

Smoothing energy. To propagate the distortion from the warping smoothly and reduce the foldover occurrence on the grid mesh and road network, a smoothing energy $D_{S m}$ is introduced into the optimization. By following the mesh smoothing concept in [29], Laplacian smoothing is adopted, which is formulated as follows:
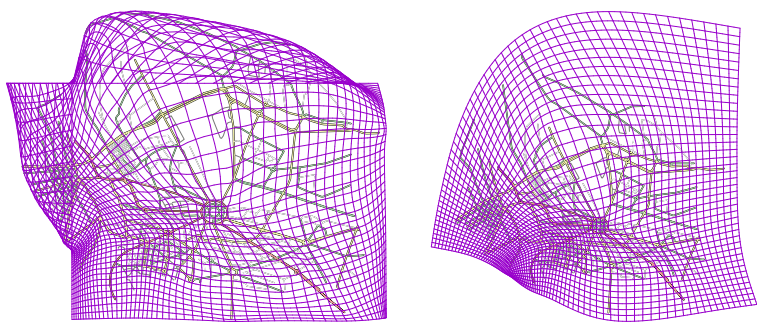

Fig. 9. Road network warping with (right) and without (left) boundary constraints. The foldover is solved by removing the boundary constraints in warping.

$$
D_{S m}(\mathbf{M})=\sum_{V \in \mathbf{V}}\|\mathbf{L} \tilde{V}\|^{2},
$$

where $\mathbf{L}$ is the uniform Laplacian matrix, which corresponds to the connectivity of the grid mesh:

$$
L_{i j}=\left\{\begin{array}{cl}
-1 / v_{v a r}, & \text { if vertices } V_{i} \text { and } V_{j} \text { are adjacent; } \\
1, & \text { if } i=j \\
0, & \text { otherwise }
\end{array}\right.
$$

$v_{v a r}$ refers to the valence of a vertex in the grid mesh. The uniform Laplacian smoothing is adopted to generate a smooth mesh. Specifically, each vertex is forced to lie in the centroid of its one-ring neighbors, thus producing a smooth deformation and greatly reducing the possibility of foldover.

Optimization of energy function. By combining all energies, the final optimization is formulated as:

$$
\arg \min _{\tilde{\mathbf{V}}}\left\{\alpha \times D_{U C}+(1-\alpha)\left(\beta \times D_{S P}+(1-\beta) \times D_{S m}\right)\right\},
$$

where $\alpha$ is the weighting factor that balances the contributions of the user-defined geometric constraints $\left(D_{U C}\right)$ and aesthetic constraints $\left(D_{S P}\right.$ and $\left.D_{S m}\right) ; \beta$ is the weighting factor of the shape-preserving term and the smoothing term. $\alpha$ is initially set to 0.8 to provide high-flexibility control for users, and $\beta$ is set to 0.5 .

In the optimization, a least-squares linear system $\mathbf{A} \tilde{\mathbf{V}}=\mathbf{b}$ with a sparse design matrix $\mathbf{A}$ can be obtained from Eq. (13). We solve this system by using an iterative solver called conjugate gradient method. The iterative process is terminated when the movements of vertices are smaller than a tolerance or the number of iterations is larger than a defined value (set to 5,000 in the experiments). To allow large deformations and reduce foldover possibility, boundary constraints that are commonly used in the route layout optimization [12], [22] are removed in our optimization. In this manner, our method can provide highly flexible warping and have a better possibility of meeting userdefined constraints. As shown in Fig. 9, the foldover problem in the warping using boundary constraints is solved by removing the boundary constraints.

A tradeoff exists between the computation cost and foldover-free guarantee because soft constraints are used in the optimization. Efficient computation is important for interactive systems, and foldover-free guarantee is necessary for map generation systems. To consider both factors, the foldover-free guarantee is set as an optional function in the current implementation. In the implementation, we check the foldover of the grid mesh after each 


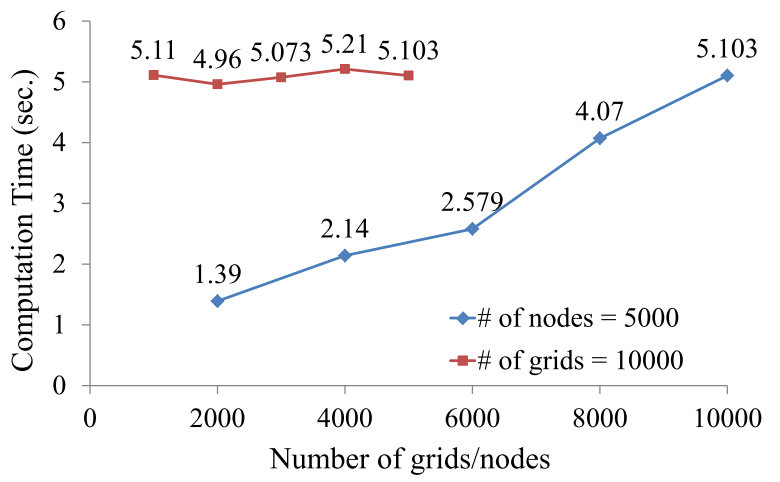

Fig. 10. Computational time of road network warping.

iteration of the optimization process. If foldover occurs, we recover the grid mesh to that in the previous iteration and increase the weight of the smoothing energy. This process is achieved by decreasing the weighting factors $\alpha$ and $\beta$ until the mesh is foldover-free. An example of foldover-free road network deformation is shown in Fig. 11. The foldover is solved by increasing the weight of the smoothing term.

\section{EXPERIMENTAL RESULtS}

Our method is tested on a PC with a $2.66 \mathrm{GHz}$ processor and 4 GB memory. For a road network containing 5,000 nodes with a grid mesh of $100 \times 100$ quads, the average computational times for warping and significance estimation are 5.1 and $0.12 \mathrm{~s}$, respectively. The computational cost of the warping depends on the number of nonzero entries in the design matrix $\mathbf{A}$ (denoted as $E$ ) and the number of iterations in the optimization solver (denoted as $T$ ). The time complexity of our mesh warping is $O(E T)$. Note that the warping computational time is independent of the number of nodes in the road network (Fig. 10) because the nodes are only used in the calculation of quad significance.

To demonstrate the shape preservation of our method, several road networks with dense structures and regular road shapes are tested. The road and quad significance values, deformed grid meshes, and road network warping results are shown in Fig. 12. All the experimental materials are available in the website (http://graphics.csie.ncku.edu. tw/Drawing-Road-Networks). In our method, high-significance roads and quads are deformed as rigidly as possible during warping. Thus, the geometric shapes and structures of significant roads can be preserved efficiently. In addition, with the aid of the smoothing energy, we can obtain smooth warping even for a large deformation on a dense road network. The results shown in Fig. 12 demonstrates the ability of our method to alleviate the foldover problem and the robustness of our method in handling large deformations on a complex road network. To test the sensitivity of the warping results to the grid mesh, various resolutions of the grid mesh are tested. The results shown in Fig. 13 indicate that a higher mesh resolution corresponds to higher warping quality but lower computational efficiency. In the system, the grid mesh resolution is a tunable parameter.

User study. A user study involving 20 participants aged 24 years old to 31 years old is conducted to evaluate the navigation utility of the generated road networks. Our evaluation tested four differently deformed road networks of Tainan, Taiwan. Each deformed road network is generated by our method and tested by five participants who are unfamiliar with the study area. The participants are required to drive to the specified destination (about $3 \mathrm{~km}$ ) with the deformed map. Thereafter, the participants fill out the questionnaire of the navigation utility in the car. The result shown in Fig. 14 indicates that these deformed maps still have the navigation function; however, Fig. $14 \mathrm{~d}$ containing the largest deformation obtains a low average

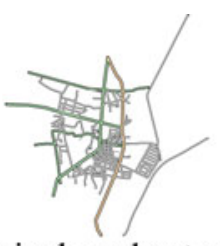

Original road network

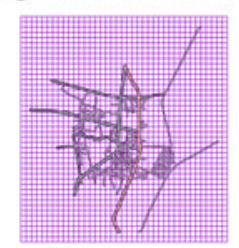

Grid mesh
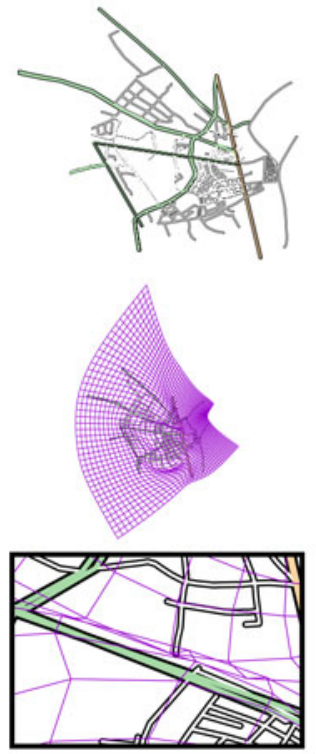

(a)
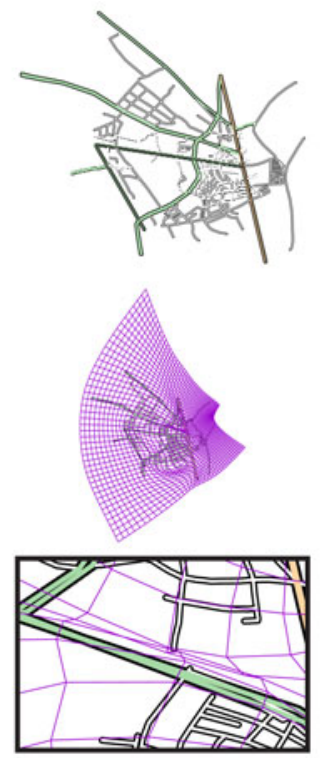

(b)
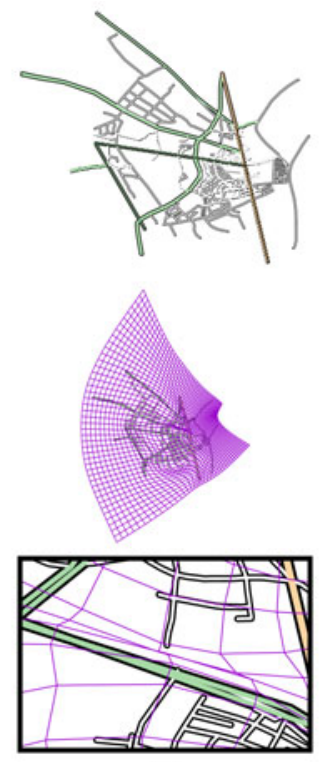

(c)
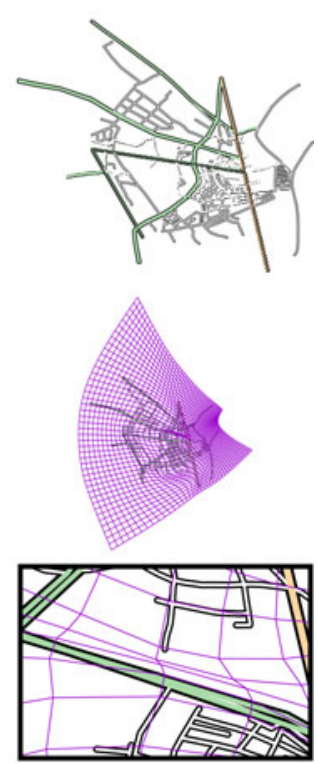

(d)

Fig. 11. Foldover-free road network warping. (a) $\alpha=0.8$ and $\beta=0.5$; (b) $\alpha=0.7$ and $\beta=0.4$; (c) $\alpha=0.6$ and $\beta=0.3$; (d) $\alpha=0.5$ and $\beta=0.2$. Left: original road network and grid mesh. Right: road network warping results (top), deformed grid meshes (middle), and close-up views of warping results (bottom). The foldovers in (a), (b), and (c) are solved by increasing the weight of the smoothing term. 


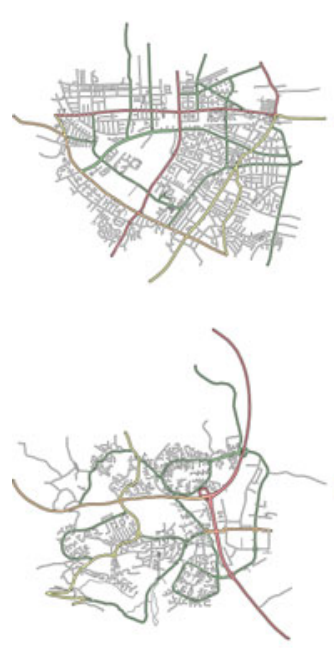

(a)
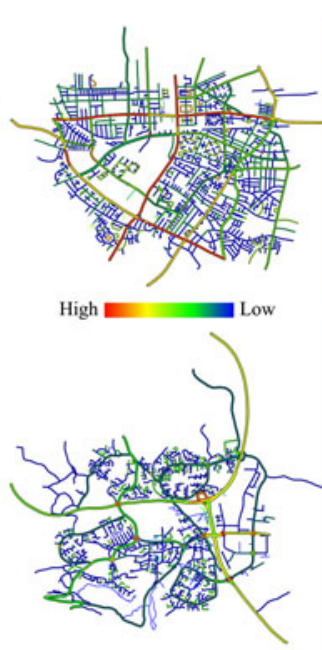

(b)
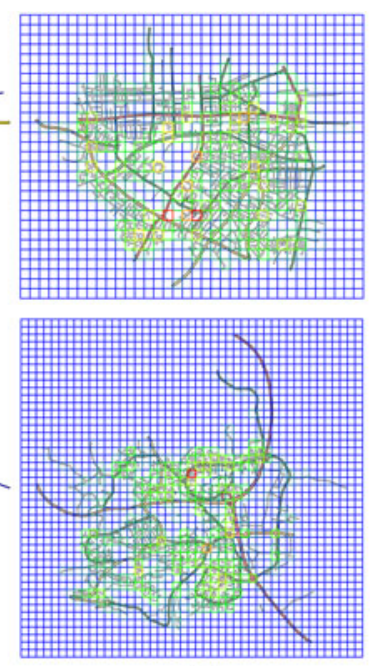

(c)

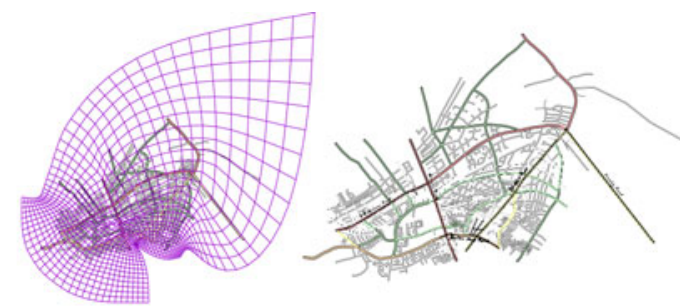

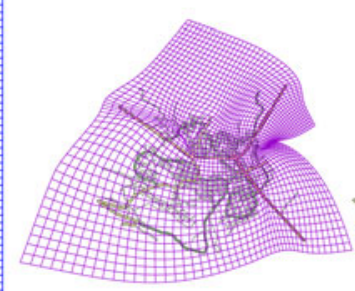

(d)

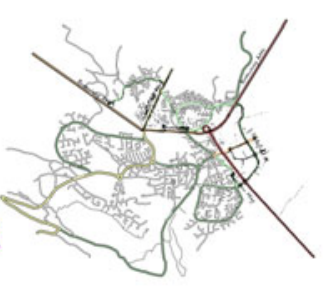

(e)

Fig. 12. Results of road network warping. (a) Original road networks; (b) road significance maps visualized by colors ranging from blue to red; (c) quad significance maps; (d) deformed grid meshes; (e) our warping results. Two warping results are shown in the top and bottom rows.

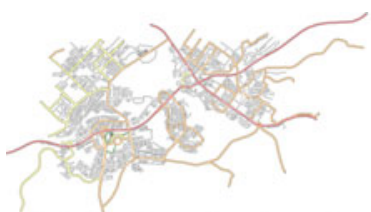

Original road network

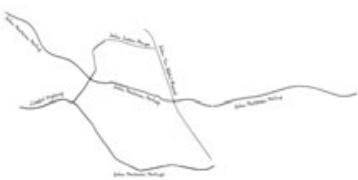

Mental map
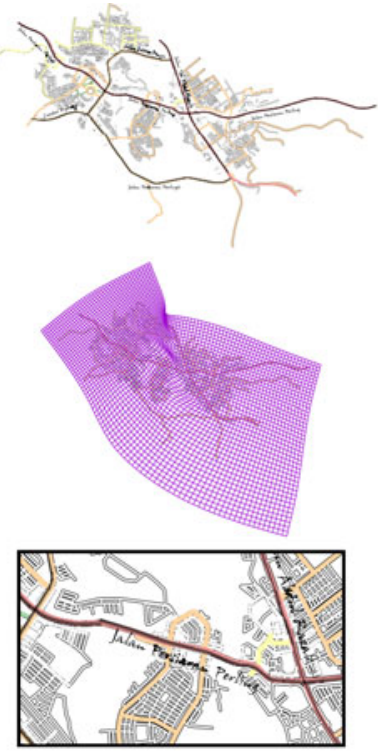

(a)
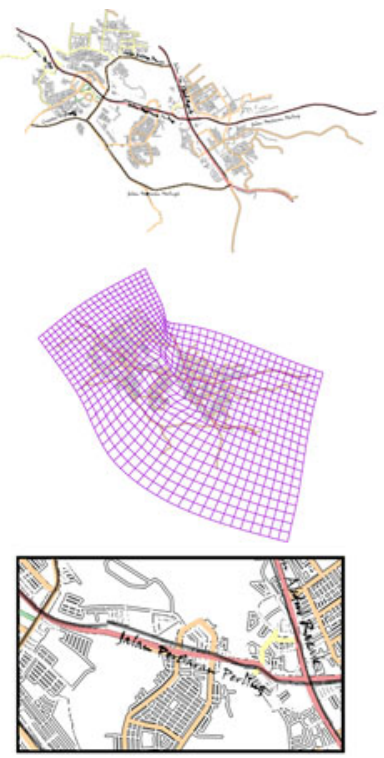

(b)
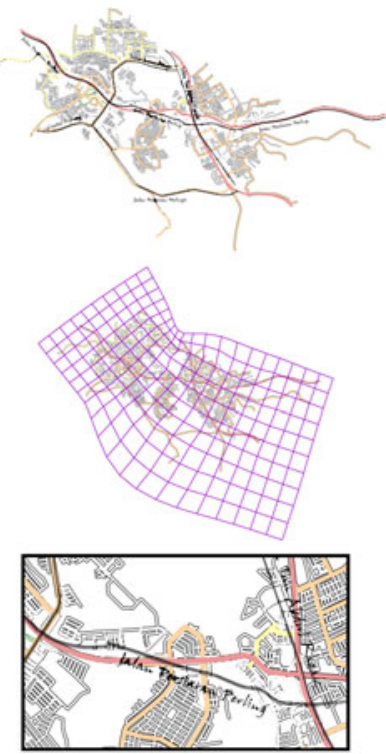

(c)

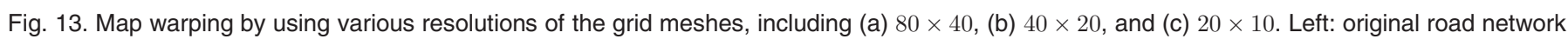
and mental map. Right: warping results (top), grid meshes (middle), and close-up views of warping results (bottom).

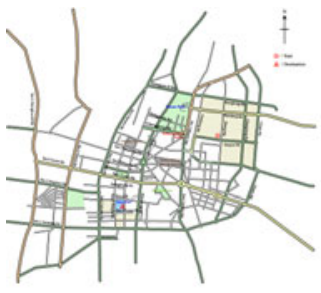

Avg. score

(a)

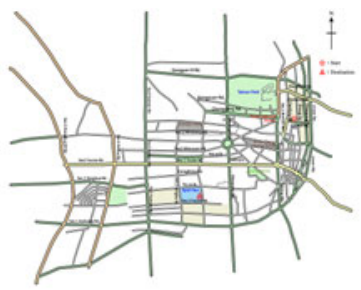

3.8

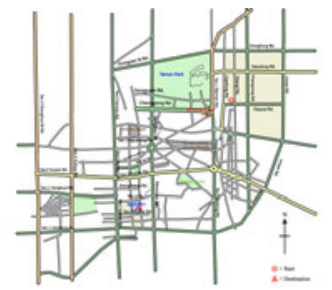

3.6

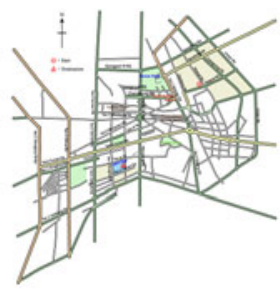

3

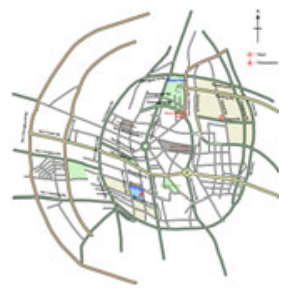

2.2

(e)

Fig. 14. User satisfaction study on navigation utility. (a) Original road network; (b), (c), (d), and (e) are deformed road networks with four user-defined constraints, respectively. Score 5: very easy to use; score 4: easy to use; score 3: fair; score 2: hard to use; score 1: very hard to use.

score, meaning that this map is not easy to use in the navigation. From this survey, we conclude that the generated maps still maintain some navigational utility because the road intersections and shapes are preserved as much as possible during warping. However, our maps with significant deformation are unsuitable for navigation. Therefore, 


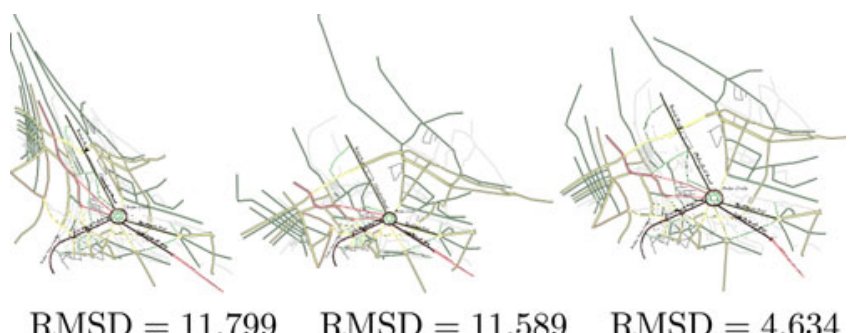

Fig. 15. Evaluation of the proposed energy terms. Left: warping without the shape-preserving term; middle: warping without the smooth term; right: warping with these terms. The original road network is shown in Fig. 3.

if an accurate navigation is required, a large deformation must be avoided in the design of the mental map. By contrast, if map advertising is the main purpose, a large deformation is allowed.

Evaluation and comparison. The root-mean-square deviation (RMSD) is used to estimate the quality of the deformed road networks. On the basis of the conclusion from the user study that user satisfaction decreases, in terms of map navigation, with increasing map deformation, the RMSD is defined according to the local deformation of the road network:

$$
\mathrm{RMSD}=\sqrt{\sum_{i=1}^{n_{\text {node }}}\left(C\left(N_{i}\right)-C\left(\tilde{N}_{i}\right)\right)^{2} / n_{\text {node }}},
$$

where $n_{\text {node }}$ represents the number of nodes in the road network, and $C\left(N_{i}\right)$ and $C\left(\tilde{N}_{i}\right)$ represent the radius of the discrete curvature of the node $N_{i}$ in the original and deformed road networks, respectively (Eq. (1)). A large RMSD indicates a large local deformation and a low map quality. To show the efficiency of the proposed shape-preserving and smoothing energy terms, our warping with and without these energy terms are evaluated by using RMSD. The warping results and RMSDs shown in Fig. 15 indicate that the shapes of the roads are preserved well with the aid of these two terms.

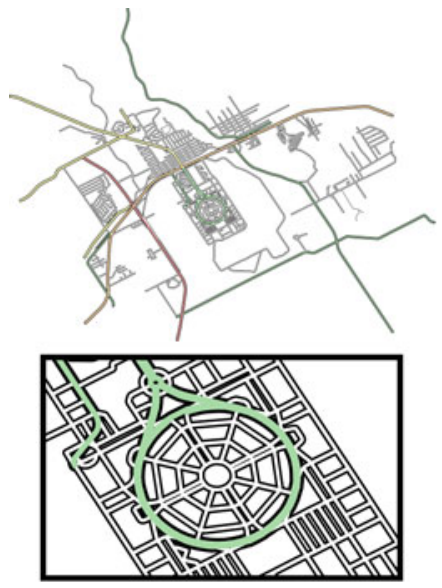

(a)

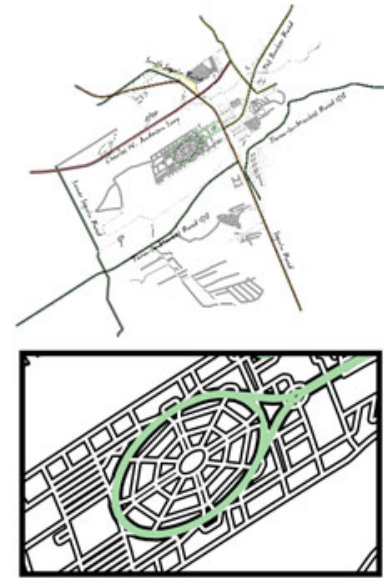

$\mathrm{RMSD}=6.547$ (b)
To evaluate the proposed warping method, our method is compared with the standard methods including feature-based warping (FBW) [30] and thin-plate spline (TPS) [31], in which user-specified line segments are used as feature pairs in [30] and corresponding nodes are used as control points in [31]. The comparison results are shown in Fig. 16. The RMSD of our result is 2.771, which is much lower than that of TPS $(\mathrm{RMSD}=4.651)$ and FBW $(\mathrm{RMSD}=6.547)$. The experimental result and also the visual comparison indicate that our results are better than those generated by these two warping methods, in terms of warping quality. For the warping methods on metro map generation [5], [10], [11], [12], map layout optimization [3], and focus region visualization [22], comparing our method with these methods is difficult because such methods are designed for optimizing the layout of a metro map or for enlarging a focus region. These methods are not designed to achieve a large deformation in a dense road network, nor conform to a user-specified mental map. For instance, the warping method presented in [22] enlarges a user-defined focus region by using a graph-based optimization, and the map layout method presented in [3] optimizes the positions, scales, and orientations of roads in a graph structure. These two methods perform well for focus region enlarging. However, without geometric constraints or without consideration to the shape preservation of road networks, these methods are unsuitable for large road network deformation.

Applications. The resulting maps from our proposed road network warping technique can serve as tourist or destination maps by highlighting representative regional or cultural marks. Some examples of destination maps are shown in Fig. 17. Three mental maps are obtained from three volunteers. The volunteers are required to sketch a map to direct the destination marked by red. The sketched maps are entered into our method as geometric constraints, and the original road network is warped to fit these maps. This application demonstrates the ability of our method to

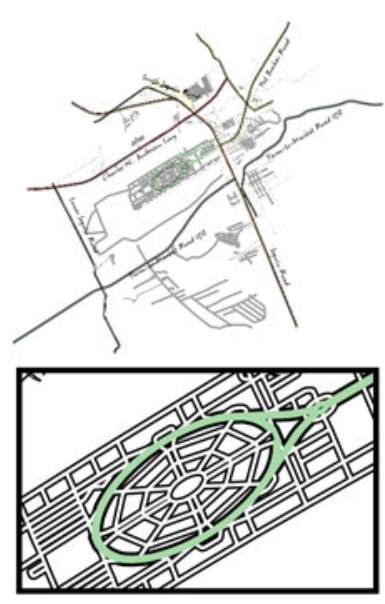

$\mathrm{RMSD}=4.651$

(c)

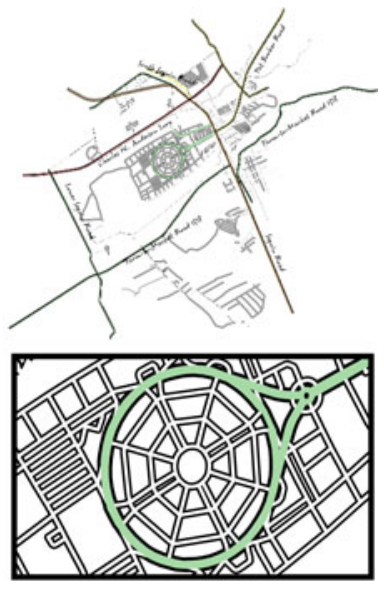

RMSD $=2.771$

(d)

Fig. 16. Comparisons between our method, FBW [30] and TPS [31]. (a) Original road network; (b) result of FBW; (c) result of TPS; (d) our results. First row: original road network and warping results; second row: close-up views. 
$x:$ The desination

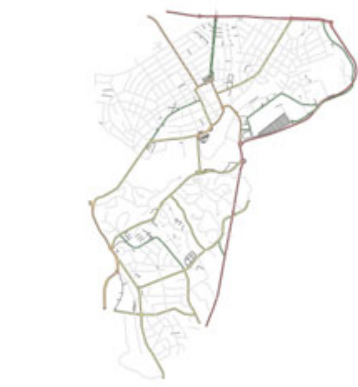

Original road network
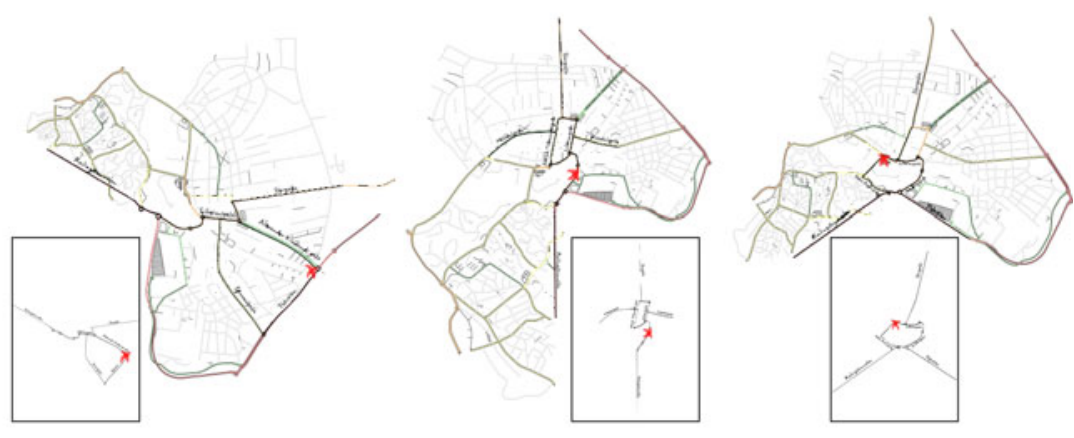

Fig. 17. Results of destination maps. Three mental maps directing a destination in Lillestrom, Norway, are created by users. The original map of this city (left figure) is deformed by using the proposed method to fit the mental maps (shown at the bottom). The resulting maps shown on the right can serve as destination maps to direct the user-specified destination.

generate a destination map by users. In Fig. 18, some deformed tourist maps generated by our method are demonstrated. The iconic marks of the tourist attractions, for example, the bird icon, which is the representative mark of New Delhi, India, and the panda icon, which is the representative mark of Chengdu, China, are integrated with the road networks. Finally, tourist maps are generated after the processes of coloring and map decoration. These examples show that our method can generate a specific shape from the road network.

Note that the generated road networks are not for GPS navigation, which requires a topographic map containing accurate information of map elements. The maps generated by our method are for people who travel in an unfamiliar area by bus or taxi. A map containing the approximate locations of destinations or tourist attractions is sufficient for tourists and visitors because such people do not really navigate the landscape.

\section{Discussion AND FUTURE WORK}

A feature-preserving road network warping with various geometric and aesthetic constraints is introduced for deformed map generation. In our warping, the road-preserving and quad-preserving constraints are used to force high-significance roads and quads to undergo asrigid-as-possible deformation, the smoothing constraint is adopted to reduce the possibility of mesh foldover, and the optimization process with the estimated significance values is used to propagate distortions. These processes efficiently ease the unpleasant deformations caused by inconsistent warping and foldover, thereby
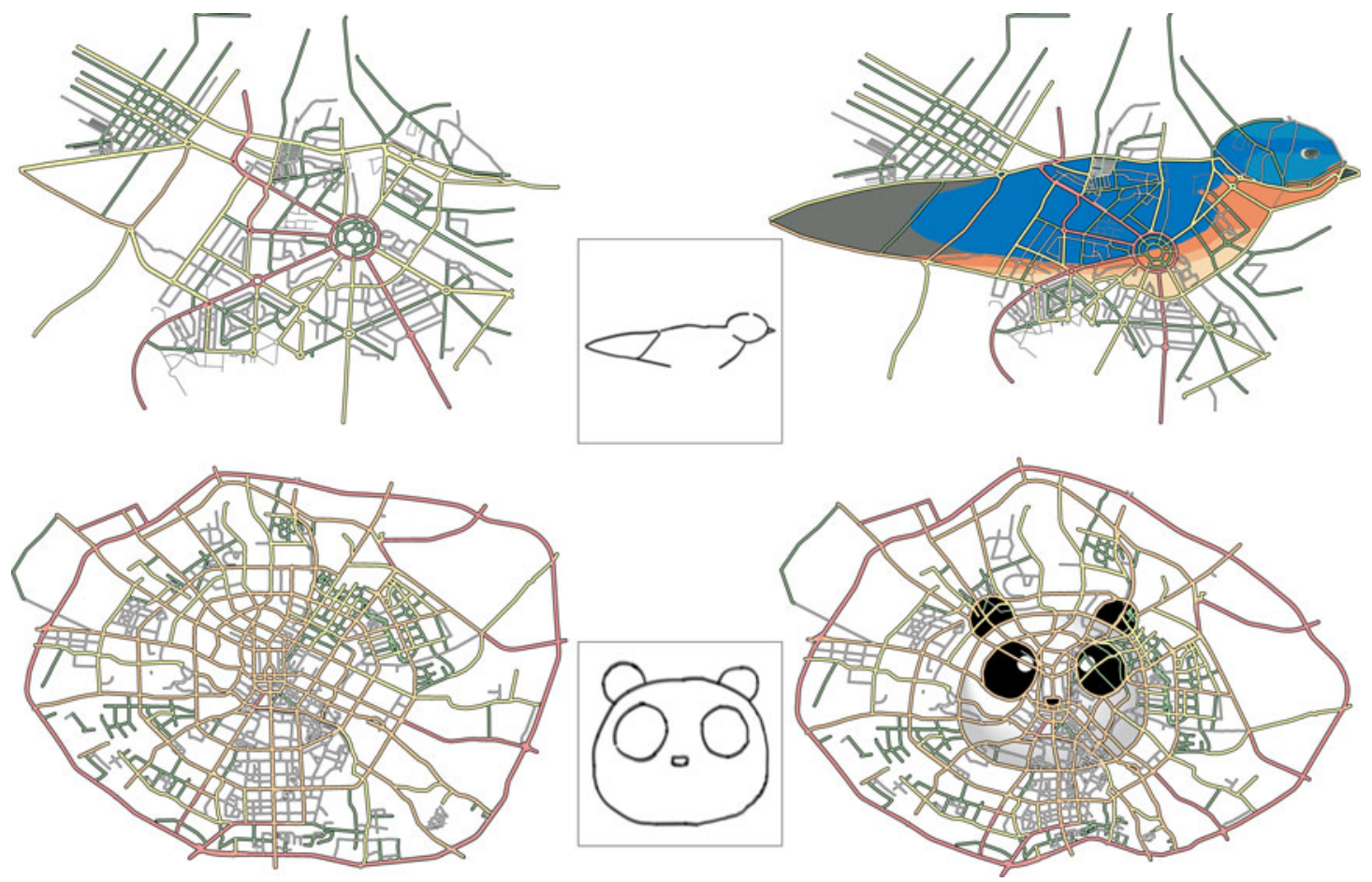

Fig. 18. Results of tourist maps. The representative iconic marks are constructed from the road networks of the tourist attractions by using our method. Thereafter, the iconic marks are colored manually in the postprocess. Top: map of New Delhi, India, which is featured with birds. Bottom: map of Chengdu, China, which is featured with pandas. The user-defined geometric constraints are shown at the middle. 
enabling our method to preserve the shapes of significant roads in a complex road network. The comparisons show the clear superiority of our method over related warping methods in terms of shape preservation. The applications on destination and tourist map generation demonstrate that our method can enable map generation system to efficiently generate various deformed maps. In the future, we plan to improve the warping performance, which is important for an interactive system. The possible solution is to reduce the number of quads in the computation of the mesh warping by using a hierarchy structure and an adaptive grid mesh. Another possible solution is to improve the solver of the least-squares system. We also plan to extend the proposed scheme to multi-layer and multi-scale maps and develop a map generation system based on our warping method and a sketch-based user interface.

\section{ACKNOWLEDGMENTS}

The authors would like to thank the anonymous reviewers for their insightful comments. This research was supported in part by the Headquarters of University Advancement at the National Cheng Kung University, and was supported in part by the National Science Council (contracts NSC-100-2628-E-006-031-MY3, NSC100-2221-E-006-188-MY3， NSC-101-2221-E-006-257-MY2, and NSC-102-2221-E-006-194), Taiwan.

\section{REFERENCES}

[1] M. Agrawala and C. Stolte, "Rendering effective route maps: Improving usability through generalization," in Proc. ACM Ann. Conf. Comput. Graph. Interactive Techn., 2001, pp. 241-249.

[2] F. Grabler, M. Agrawala, R. W. Sumner, and M. Pauly, "Automatic generation of tourist maps," ACM Trans. Graphics, vol. 27, no. 3, pp. 100:1-100:11, 2008.

[3] J. Kopf, M. Agrawala, D. Bargeron, D. Salesin, and M. Cohen, "Automatic generation of destination maps," ACM Trans. Graphics, vol. 29, no. 6, pp. 158:1-158:12, 2010.

[4] S. Avelar and L. Hurni, "On the design of schematic transport maps," Cartographica, vol. 41, no. 3, pp. 217-228, 2006.

[5] J. Böttger, U. Brandes, O. Deussen, and H. Ziezold, "Map warping for the annotation of metro maps," IEEE Comput. Graph. Appl., vol. 28, no. 5, pp. 56-65, Sept. 2008.

[6] M.M. Silvania Avelar, "Generating topologically correct schematic maps," in Proc. 9th Int. Symp. Spatial Data Handling, 2000, pp. 4-28.

[7] J. Krygier and D. Wood, Making Maps: A Visual Guide to Map Design for GIS. New York, NY, USA: Guilford Press, 2005.

[8] M.S.T. Carpendale, D.J. Cowperthwaite, and F.D. Fracchia, "3dimensional pliable surfaces: For the effective presentation of visual information," in Proc. 8th Annu. ACM Symp. User Interface Softw. Technol., 1995, pp. 217-226.

[9] T.A. Keahey and E.L. Robertson, "Techniques for non-linear magnification transformations," in Proc. IEEE Symp. Inf. Vis., 1996, pp. 38-45.

[10] M. Nöllenburg and A. Wolff, "Drawing and labeling high-quality metro maps by mixed-integer programming," IEEE Trans. Vis. Comput. Graph., vol. 17, no. 5, pp. 626-641, May 2011.

[11] J. Stott, P. Rodgers, J.C. Martinez-Ovando, and S.G. Walker, "Automatic metro map layout using multicriteria optimization," IEEE Trans. Vis. Comput. Graph., vol. 17, no. 1, pp. 101-114, Jan. 2011.

[12] Y.-S. Wang and M.-T. Chi, "Focus+context metro maps," IEEE Trans. Vis. Comput. Graph., vol. 17, no. 12, pp. 2528-2535, Dec. 2011.

[13] M. Denis, "The description of routes: A cognitive approach to the production of spatial discourse," Cahiers Psychologie Cognitive, vol. 16, no. 4, pp. 409-458, 1997.
[14] A.M. MacEachren, How Maps Work: Representation, Visualization, and Design. New York, NY, USA: Guilford Press, 1995.

[15] L. Harrie, "An optimisation approach to cartographic generalisation," Doctoral thesis, Dept. Surveying, Lund Inst. Technol., Lund Univ. (Schweden), 2001.

[16] I. Simon, N. Snavely, and S.M. Seitz, "Scene summarization for online image collections," in Proc. 11th IEEE Int. Conf. Comput. Vision, 2007, pp. 1-8.

[17] W.-C. Chen, A. Battestini, N. Gelfand, and V. Setlur, "Visual summaries of popular landmarks from community photo collections," in Proc. 17th ACM Int. Conf. Multimedia, 2009, pp. 789-792.

[18] N. Snavely, S.M. Seitz, and R. Szeliski, "Photo tourism: Exploring photo collections in 3d," ACM Trans. Graph., vol. 25, no. 3, pp. 835-846, 2006.

[19] T. Isenberg, "Visual abstraction and stylisation of maps," Cartographic J., vol. 50, pp. 8-18, 2013.

[20] S. Afzal, R. Maciejewski, Y. Jang, N. Elmqvist, and D.S. Ebert, "Spatial text visualization using automatic typographic maps," IEEE Trans. Vis. Comput. Graph., vol. 18, no. 12, pp. 2556-2564, Dec. 2012.

[21] Y.-S. Wang, T.-Y. Lee, and C.-L. Tai, "Focus+context visualization with distortion minimization," IEEE Trans. Vis. Comput. Graph., vol. 14, no. 6, pp. 1731-1738, Nov./Dec. 2008.

[22] J.-H. Haunert and L. Sering, "Drawing road networks with focus regions," IEEE Trans. Vis. Comput. Graph., vol. 17, no. 12, pp. 25552562, Dec. 2011.

[23] S.-S. Lin, C.-H. Lin, I.-C. Yeh, S.-H. Chang, C.-K. Yeh, and T.-Y. Lee, "Content-aware video retargeting using object-preserving warping," IEEE Trans. Vis. Comput. Graph., vol. 19, no. 10, p. 16771686, Oct. 2013.

[24] M.A. Cobb, M.J. Chung, H. Foley, F.E. Petry, K.B. Shaw, and H.V. Miller, "A rule-based approach for the conflation of attributed vector data," GeoInformatica, vol. 2, pp. 7-35, 1998.

[25] G. Touya, A. Coupe, J.L. Jollec, O. Dorie, and F. Fuchs, "Conflation optimized by least squares to maintain geographic shapes," ISPRS Int. J. Geo-Inf., vol. 2, no. 3, pp. 621-644, 2013.

[26] V. Eppell, J. Bunker, and B. McClurg, "A four level road hierarchy for network planning and management," in Proc. 20th Australian Road Res. Board Conf., 2001, pp. 1-15.

[27] B. Tversky, "Distortions in memory for maps," Cognitive Psychology, vol. 13, no. 3, pp. 407-433, 1981.

[28] B. Jiang and C. Claramunt, "A structural approach to the model generalization of an urban street network," Geoinformatica, vol. 8, no. 2, pp. 157-171, 2004.

[29] A. Nealen, T. Igarashi, O. Sorkine, and M. Alexa, "Laplacian mesh optimization," in Proc. 4th Int. Conf. Comput. Graph. Interactive Techn. Australasia Southeast Asia, 2006, pp. 381-389.

[30] T. Beier and S. Neely, "Feature-based image metamorphosis," in Proc. ACM 19th Ann. Conf. Comput. Graph. Interactive Techn., 1992, pp. 35-42.

[31] F.L. Bookstein, "Principal warps: Thin-plate splines and the decomposition of deformations," IEEE Trans. Pattern Anal. Mach. Intell., vol. 11, no. 6, pp. 567-585, June 1989.

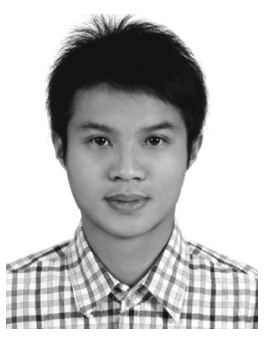

Shih-Syun Lin received the BS degree in applied mathematics from Providence University, Taiwan in 2007 and the MS degree from Graduate Institute of Educational Measurement and Statistics from National Taichung University, Taiwan in 2010. Currently, he is working toward the $\mathrm{PhD}$ degree in the Department of Computer Science and Information Engineering, National Cheng-Kung University, Taiwan. His research interests include video retargeting, mesh deformation, pattern recognition, and computer graphics. 


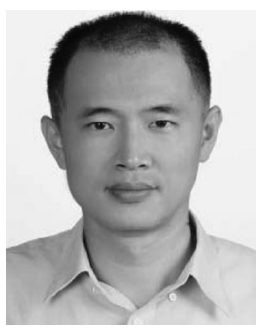

Chao-Hung Lin received the $\mathrm{MS}$ and $\mathrm{PhD}$ degrees in computer science and information engineering from National Cheng-Kung University, Taiwan in 1998 and 2004, respectively. Since 2006, he has been a member of the faculty of the Department of Geomatics at National Cheng-Kung University. He is currently an associate professor. He leads the Digital Geometry Processing Laboratory (http://dgl.geomatics. ncku.edu.tw) and co-leads the Computer Graphics Laboratory, National Cheng-Kung University (http://graphics.csie.ncku.edu.tw). His current research interests include remote sensing, point cloud processing, digital map generation, information visualization, and computer graphics. He served as an editorial board member of the International Journal of Computer Science and Artificial Intelligence. He is a member of the IEEE and the ACM.

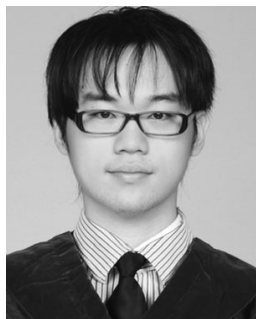

Yan-Jhang Hu received the BS degree from the Department of Information and Computer Engineering, Chung Yuan Christian University, Taiwan in 2011 and the MS degree from the Department of Computer Science and Information Engineering, National Cheng-Kung University, Taiwan in 2013. Currently, he is with Acer Inc. His research interest is computer graphics.

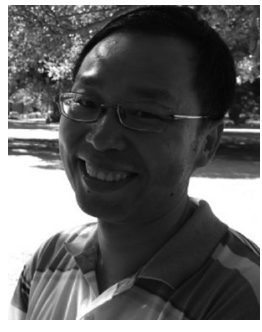

Tong-Yee Lee received the $\mathrm{PhD}$ degree in computer engineering from Washington State University, Pullman, in May 1995. He is currently a chair professor in the Department of Computer Science and Information Engineering, National Cheng-Kung University, Tainan, Taiwan, ROC. He leads the Computer Graphics Group, Visual System Laboratory, National Cheng-Kung University (http://graphics.csie.ncku.edu.tw/). His current research interests include computer graphics, nonphotorealistic rendering, medical visualization, virtual reality, and media resizing. He is a senior member of the IEEE and a member of the ACM.

$\triangleright$ For more information on this or any other computing topic, please visit our Digital Library at www.computer.org/publications/dlib. 